\title{
Tuning carrier density across Dirac point in epitaxial graphene on SiC by corona discharge
}

\author{
Arseniy Lartsev, ${ }^{1}$ Tom Yager, ${ }^{1}$ Tobias Bergsten, ${ }^{2}$ Alexander Tzalenchuk, ${ }^{3}$ T.J.B.M Janssen, ${ }^{3}$ Rositza \\ Yakimova, ${ }^{4,5}$ Samuel Lara-Avila, ${ }^{1}$ and Sergey Kubatkin ${ }^{1}$ \\ ${ }^{1)}$ Department of Microtechnology and Nanoscience, Chalmers University of Technology, S-41296 Göteborg, \\ Sweden \\ 2) SP Technical Research Institute of Sweden, S-50115 Borås, Sweden \\ 3) National Physical Laboratory, TW110LW, Teddington, UK \\ ${ }^{4)}$ Department of Physics, Chemistry and Biology (IFM), Linköping University, S-58183 Linköping, \\ Sweden \\ ${ }^{5)}$ Graphensic AB, SE-58330 Linköping, Sweden
}

We demonstrate reversible carrier density control across the Dirac point $\left(\Delta n \sim 10^{13} \mathrm{~cm}^{-2}\right)$ in epitaxial graphene on $\mathrm{SiC}(\mathrm{SiC} / \mathrm{G})$ via high electrostatic potential gating with ions produced by corona discharge. The method is attractive for applications where graphene with a fixed carrier density is needed, such as quantum metrology, and more generally as a simple method of gating 2DEGs formed at semiconductor interfaces and in topological insulators.

Keywords: Epitaxial graphene, corona discharge, quantum Hall effect

Graphene, discovered in $2004^{1}$, is rapidly emerging as a revolutionizing material for the enhancement or development of novel electronic devices. ${ }^{2}$ In view of applications, an industrially relevant way to produce highquality graphene on wafer scale is the thermal decomposition of silicon carbide ( $\mathrm{SiC})$ at high temperatures. ${ }^{3} \mathrm{In}$ particular, graphene grown on the $\mathrm{Si}$-face of $\mathrm{SiC}(\mathrm{SiC} / \mathrm{G})$ has demonstrated its enormous potential in electronics by being the material of choice for the fabrication of graphene-based high performance electronic devices such as high-frequency analogue transistors ${ }^{4}$, sensors, ${ }^{5}$ and quantum resistance standard? . $^{\text {. }}$

In $\mathrm{SiC} / \mathrm{G}$ the two-dimensional system is formed by graphene situated on top of a non-conducting buffer carbon layer (0-layer), covalently bonded to SiC. Charge transfer between graphene and the localised donor states in the buffer, driven by the work function difference $A \sim 1 \mathrm{eV}$, leads to a significant electron density in the as grown $\mathrm{SiC} / \mathrm{G}$, typically $n \sim 10^{13} \mathrm{~cm}^{-2}{ }^{6}$ This charge transfer leads to a robust quantum Hall quantization, making this material a superior embodiment of the quantum resistance standard compared to conventional GaAs heterostructures. ${ }^{7,8}$ Charge transfer from the substrate manifests in quantum magnetotransport as anomalously wide quantum Hall plateux and high breakdown currents that have lead to the most accurate resistance quantisation measurements to-date. ${ }^{8}$

The ideal technique for carrier density control would preserve the rich interplay between the graphene layer and the substrate. The standard GFET configuration applied to epitaxial graphene shows promise for transistor applications, but, as far as precision metrology is concerned, suffers from unacceptable gate dielectric leakage, up to nanoamperes per square micrometre of the gate area, even at modest voltages of a few volt. ${ }^{9}$

A possible approach to carrier density control of $\mathrm{SiC} / \mathrm{G}$ is the intercalation of gases such as hydrogen ${ }^{10}$ or oxygen ${ }^{11}$ at the graphene/SiC interface, with the fur- ther possibility to ion-implant a bottom gate. ${ }^{12}$ However, these techniques suffer from instability under ambient or high temperature conditions and more importantly, suppress the $\mathrm{SiC} / \mathrm{G}$-substrate interaction limiting their use in quantum metrology applications. Other carrier density control methods which preserve the graphene$\mathrm{SiC}$ charge transfer include chemical doping of graphene by non-covalent functionalization ${ }^{13}$ and photochemical gating. ${ }^{14}$ Drawbacks of chemical doping are instability in ambient conditions and degradation of carrier mobility due to the introduction of charged scatterers. In photochemical gating the photo-generated dopants are spatially decoupled from the graphene to preserve its carrier mobility. This technique proved effective for gating in the range $\Delta n \sim 10^{12} \mathrm{~cm}^{-2}$ and was instrumental in the success of the quantum resistance metrology work. However it is insufficient to achieve charge neutrality or charge inversion in most as-grown $\mathrm{SiC} / \mathrm{G}$ samples.

Here we present an alternative approach for reversible tuning of the carrier density of $\mathrm{SiC} / \mathrm{G}$ in a range $\sim 10^{13}$ carriers $\mathrm{cm}^{-2}$ by ions generated by corona discharge. ${ }^{15}$ Using this method we have been able to control the doping levels of large area $\left(\sim \mathrm{mm}^{2}\right) \mathrm{SiC} / \mathrm{G}$ so that charge neutrality and p-doping were achieved in initially strongly n-doped devices. Gating by corona discharge has been widely used in semiconductor industry to control carrier concentration in oxidized semiconductors in a contact-less fashion. ${ }^{16-19}$ In this technique the metallic gate of a FET is replaced by ions deposited on the dielectric layer, which induce a surface charge density on the underlying semiconductor. A significant advantage of using corona ions instead of the metallic top gate is the larger range over which the carrier concentration can be tuned, due to the low mobility of the ions in the dielectric which suppresses the effect of dielectric breakdown ${ }^{19}$.

The devices used in this study are Hall bars fabricated on $\mathrm{SiC} / \mathrm{G}$ grown on Si-face at $T=2000^{\circ} \mathrm{C}$ and $P=1$ atm Ar (Graphensic AB). ${ }^{3}$ Hall bars of 
a)

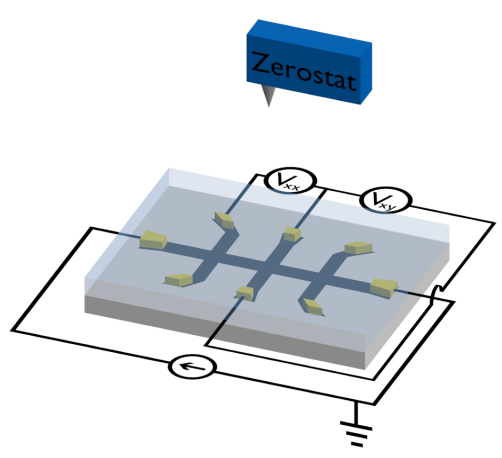

c)

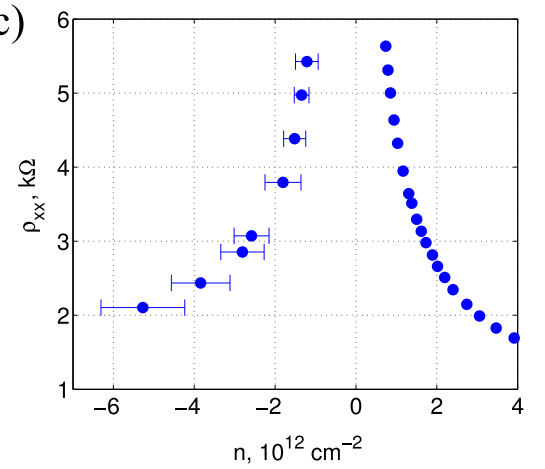

b)

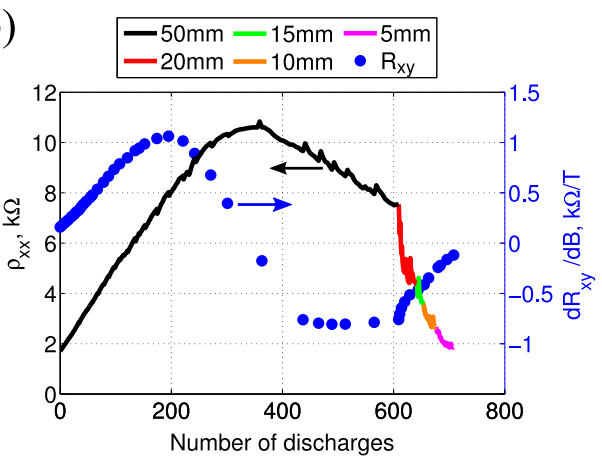

d)

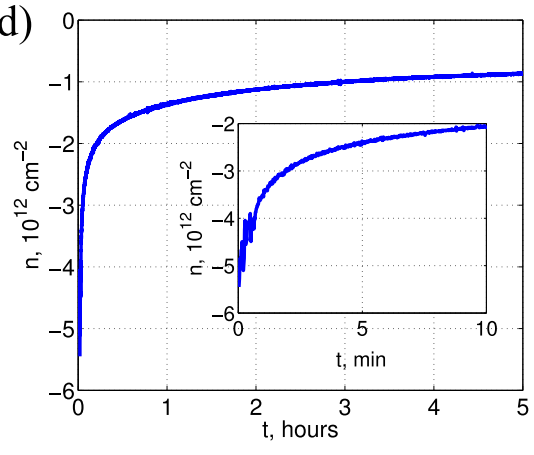

FIG. 1. Controlling carrier density in a SiC/G Hall bar $(30 \times 180 \mu \mathrm{m})$ by ions generated using corona discharge in ambient conditions. a) A sketch of the measurement setup. The evolution of the longitudinal resistance $R_{x x}$ was measured in real-time as the sample was exposed to ions. b) Evolution of $\rho_{x x}=R_{x x} W / L$ and the Hall coefficient $R_{H}=d R_{x y} / d B$ as a function of the number of ion discharges, with different distance between the ion source and the sample. The Dirac point (maximum in $\rho_{x x}$ ) is reached after 375 discharges at the distance of $50 \mathrm{~mm}$. c) Longitudinal resistivity $\rho_{x x}$ as a function of carrier concentration $n$. The increased uncertainty of $R_{x y}(B)$ for p-type doping is due to a faster drift of carrier density observed at high hole concentration. d) Time evolution (drift) of carrier density after the maximum p-doping of $\sim 5 \cdot 10^{12}$ was reached. All measurements taken at room temperature.

different dimensions were patterned on $\mathrm{SiC} / \mathrm{G}$ using standard electron-beam lithography, lift-off and oxygen plasma etching, as reported elsewhere. ${ }^{20}$ The sample was then spin-coated with a thin layer $(50,100$ or $400 \mathrm{~nm})$ of poly(methy lmethacrylate- co -methacrylate acid), henceforth P(MMA-MAA) (MicroChem Corp., PMMA copolymer resist solids $6 \%$ in ethyl lactate). For this letter we focus on two Hall bars: device A with dimensions $30 \times 180 \mu \mathrm{m}^{2}$ and millimetre-sized $\left(1.25 \times 2.5 \mathrm{~mm}^{2}\right)$ device B. All results reported in this work were obtained with $100 \mathrm{~nm}$ thick layer; room temperature measurements were repeated for $50 \mathrm{~nm}$ and $400 \mathrm{~nm}$ thick layer but no significant effect of polymer thickness was observed.

Carrier density control of $\mathrm{Si} / \mathrm{C}$ samples was performed in ambient conditions employing a piezo-activated antistatic gun to generate the corona discharge. The device produced a $1-2 \mathrm{~s}$ long pulse of positive or negative ions with the polarity determined by the sign of deformation (compression or expansion) of the piezo-crystal.

Changes in the electronic properties of $\mathrm{SiC} / \mathrm{G}$ upon exposure to corona ions were monitored in real time through continuous measurements of the longitudinal resistance $R_{x x}$ and sampling of the transversal resistance $R_{x y}$. The measurement setup is shown in 1a; the Hall bar under study was connected to a multimeter and grounded at all time.

Corona ions allow reversible tuning of the carrier density in $\mathrm{SiC} / \mathrm{G}$ in a range of $\sim 10^{13} \mathrm{~cm}^{-2}$. The evolution of the longitudinal resistivity $\rho_{x x}=R_{x x} W / L$ and the Hall coefficient $R_{H}=d R_{x y} / d B$ as a function of number of ion pulses for device $\mathrm{A}$ is displayed in $1 \mathrm{~b}$. The carrier density in this device was changed from the initial n-doping of $4 \cdot 10^{12} \mathrm{~cm}^{-2}$ to p-doping at the level of $5 \cdot 10^{12} \mathrm{~cm}^{-2}$. This was achieved by exposing the sample to repeated ion pulses with a time interval of $5-20 \mathrm{~s}$. As a function of the number of pulses, the longitudinal resistivity first gradually increased to a maximum value of $\sim 11 \mathrm{k} \Omega$ and then monotonically decreased. By comparing $\rho_{x x}$ with the measured carrier density (1c), we confirmed that the observed maximum in the longitudinal resistivity corresponds to the Dirac point, signalling the cross-over from n- to p-doping. As a remark, we observed that for each ion pulse, a rapid change of $\rho_{x x}$ was followed by a steady drift in the opposite direction due to charge leakage. This drift rate increased as the total change in doping was increasing, shown in $1 \mathrm{~d}$.

The sensitivity of carrier density in $\mathrm{SiC} / \mathrm{G}$ to ion pulses depends strongly on the distance between the sample and 
a)

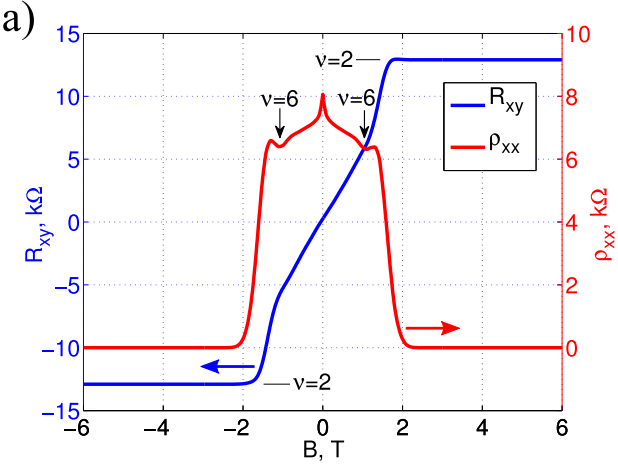

b)

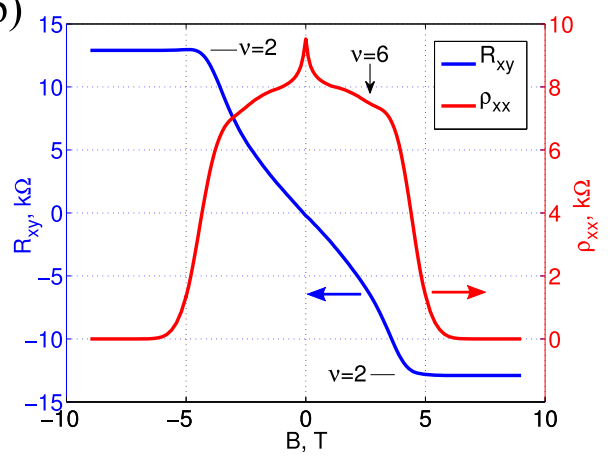

FIG. 2. Low temperature magnetotrasport on $\mathrm{SiC} / \mathrm{G}$ exposed to corona ions. (a) Half-integer quantum Hall effect observed for electron doping reveals the monolayer nature of device $\mathrm{A}$ $(30 \times 180 \mu \mathrm{m})$ at $\mathrm{T}=2 \mathrm{~K}$. (b) QHE in the same device for p-doping, signalled by the negative slope in $R_{x y}(B)$.

the corona source. For example, the effect of discharge ions generated $50 \mathrm{~mm}$ from the sample saturated after 600 cycles, when the carrier concentration reached a hole density of $\sim 5 \cdot 10^{11} \mathrm{~cm}^{-2}$. At this point addition of more negative static charge was compensated by charge leakage. Nonetheless, the hole density could be further increased to $5 \cdot 10^{12} \mathrm{~cm}^{-2}$ by decreasing the source-sample distance to $5 \mathrm{~mm}$ (1b). From that point and with no additional ion pulses the doping concentration drifted back (1d), first quickly and then slower, reaching the charge neutrality point after 24 hours and the electron density of $8 \cdot 10^{11} \mathrm{~cm}^{-2}$ after 7 days. It was also possible to accelerate this backwards drift and even overcompensate the initial electron density by generating positive corona ions.

The wide carrier density tuning range enabled by corona ions allows complete electronic characterization of $\mathrm{SiC} / \mathrm{G}$ samples via low temperature magnetotransport measurements. That is, the heavy intrinsic n-doping of $\mathrm{SiC} / \mathrm{G}$ can be decreased close to charge neutrality (and beyond, to p-doping) to allow the appearance of halfinteger quantum Hall effect (hi-QHE) at experimentally accessible magnetic fields. With the carrier density tuned to electron doping at the level $1.3 \cdot 10^{11} \mathrm{~cm}^{-2}$ the device A showed the Hall mobility of $6000 \mathrm{~cm}^{2} / \mathrm{V} \cdot \mathrm{s}$, the $\nu=2$ plateau starting at $2 \mathrm{~T}$, and a clear signature of $\nu=6$ in $\rho_{x x}(B)(2 \mathrm{a})$. The sample was subsequently warmed a)

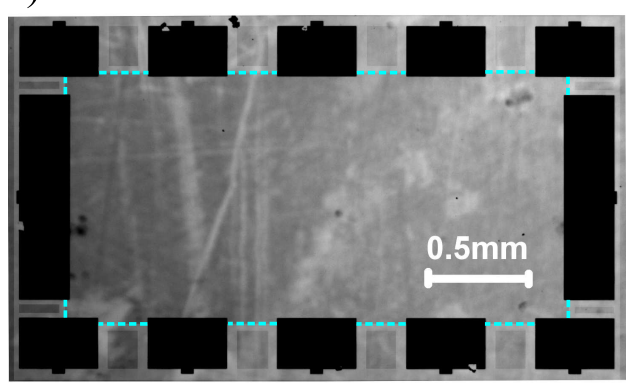

b)

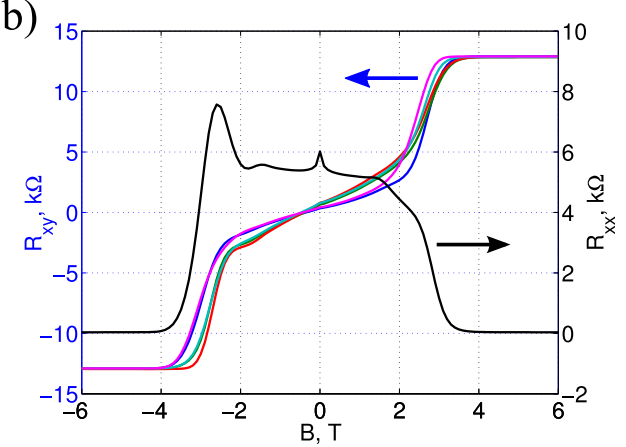

FIG. 3. Carrier density control by corona ions in milimetresized device B $(1.25 \times 2.5 \mathrm{~mm})$ : a) Optical microscope (transmission) image of the device; the electric contacts appear as dark rectangles, edge of the graphene device is highlited. b) low temperature magneto transport measurements showing onset of half-integer quantum Hall plateaux in $R_{x y}$ at magnetic field $B \sim 4 \mathrm{~T}$ for different pairs of Hall probes.

up to room temperature and exposed further to negative corona ions until $\rho_{x x}$ passed its maximum at the Dirac point. Magnetotransport measurements were performed on the p-doped device at $\mathrm{T}=2 \mathrm{~K}$, showing once again the $\nu=2$ plateau and a faint signature of $\nu=6$, carrier density $3.3 \cdot 10^{11} \mathrm{~cm}^{-2}$ and mobility $2000 \mathrm{~cm}^{2} / \mathrm{V} \cdot \mathrm{s}(2 \mathrm{~b})$. As a remark, the characteristic time over which carrier density drifts is on the order of minutes at room temperature, but this drift stops when the sample is cooled down to a temperature $T \lesssim 200 \mathrm{~K}$. Therefore, once the desired carrier concentration is achieved at room temperature the sample is loaded into the cryostat and we have observed stability of carrier density at low temperatures throughout our experiments lasting weeks.

Without further optimization of the set-up geometry, the homogeneity of doping by corona ions was tested on milimetre-sized $\left(1.25 \times 2.5 \mathrm{~mm}^{2}\right)$ device B (3). The corona source, a needle with diameter $0.5 \mathrm{~mm}$ and tip radius of the order of $50 \mu \mathrm{m}$, was placed $20 \mathrm{~mm}$ from this device. By discharging negative ions on this device the electron density was tuned in the range of $3-5 \cdot 10^{11} \mathrm{~cm}^{-2}$ and mobility of about $5800 \mathrm{~cm}^{2} / \mathrm{V} \cdot \mathrm{s}$ was measured. These parameters allowed observation of QHE at magnetic fields below $B=5 \mathrm{~T}$ with a quantization precision of at least $4 \cdot 10^{-5}$, limited by the measure- 
ment setup.

The low mobility of ionic species in the dielectric allows the formation of large electric fields, causing big charge in doping. Since the graphene device under study was grounded, negative static charge in the polymer layer induced an equal amount of positive charge on the surface of $\mathrm{SiC} / \mathrm{G}$. The maximum achieved change in doping was from $n_{1} \approx 4 \cdot 10^{12} \mathrm{~cm}^{-2}$ to $n_{2} \approx-5 \cdot 10^{12} \mathrm{~cm}^{-2}$ (negative carrier density indicates hole doping), and equivalent density of singly charged negative ions in the polymer is $n_{g}=-\int_{n_{1}}^{n_{2}} d n / r_{d}(n)$ where $r_{d}(n)=-d n / d n_{g}$ is gate responsivity depending on $n$. In $\mathrm{SiC} / \mathrm{G}, r$ is less than 1 due to charge transfer between graphene and the substrate. Solving the equation for the charge balance between graphene and surface donor states in the buffer layer 6

$$
\gamma\left(A-\frac{e^{2} d}{\epsilon_{0}}\left(n+n_{g}\right)-\epsilon_{f}(n)\right)=n+n_{g}
$$

where $\gamma$ is the density of the donor states, $d$ is the distance between the graphene sheet and the donor states, and $\epsilon_{f}(n)=\hbar v_{f} \sqrt{\pi} \cdot \operatorname{sgn}(n) \cdot \sqrt{|n|}$ is the Fermi level in graphene, we obtain the gate responsivity in the form

$$
r_{d}=\left(1+\frac{\gamma \hbar v_{f} \sqrt{\pi}}{1+\gamma \frac{e^{2} d}{\epsilon_{0}}} \cdot \frac{1}{2 \sqrt{|n|}}\right)^{-1}
$$

For example, assuming $d \approx 0.3 \mathrm{~nm}$ and $\gamma \sim 10^{13} \mathrm{eV}^{-1}$ $\mathrm{cm}^{-26}$, this gives $r_{d}\left(n_{1}\right) \approx r_{d}\left(n_{2}\right) \approx 0.85$ and $n_{g} \approx 1.2$. $10^{13} \mathrm{~cm}^{-2}$.

The electric field inside the polymer can be calculated as $E=e n_{g} / \epsilon \epsilon_{0}$ where $\epsilon \sim 4$ is the dielectric constant of $\mathrm{P}\left(\right.$ MMA-MAA). Assuming the above estimate $n_{g} \approx 1.2$. $10^{13} \mathrm{~cm}^{-2}$, this gives $E \sim 500 \mathrm{MV} / \mathrm{m}$. This is a rather high field considering that PMMA is typically reported to show leakage current on the level of $10^{-6} \mathrm{~A} / \mathrm{cm}^{2}$ already at $100 \mathrm{MV} / \mathrm{m}^{21,22}$ while in our case, leakage current can be estimated as $j=\frac{e}{r} \cdot d n / d t \sim 10^{-8} \mathrm{~A} / \mathrm{cm}^{2}$. This indicates that the corona gate is indeed less prone to leakage.

As for the maximum doping range attainable with corona ions in $\mathrm{SiC} / \mathrm{G}$, our observations support the hypothesis that the limit is set by the leakage current and not by the fact that accumulated charge starts to repel incoming ions. The potential difference between graphene and polymer surface can be estimated as $V \sim E \cdot d$, which for a $100 \mathrm{~nm}$ thick polymer layer gives $V \sim 50 \mathrm{~V}$, much less than the voltage of corona discharge (typically a few $\mathrm{kV}$ ). Two possible mechanisms of charge leakage are charge transfer through the polymer and leakage into the ambient. We therefore expect that the leakage current depends mainly on the electric field in the polymer and/or density of charge on its surface, but not on the thickness of the polymer. This is in agreement with our observation that the maximum change in doping didn't show any significant dependence on polymer thickness.

In conclusion, we have demonstrated tuning of carrier density in the range of $\sim 10^{13}$ carriers $\cdot \mathrm{cm}^{-2}$ in epitax- ial graphene on silicon carbide using corona discharge. This allowed us to achieve both low carrier densities $\left(\sim 10^{11} \mathrm{~cm}^{-2}\right)$ and strong p-type doping (up to $5 \cdot 10^{12}$ holes $\left.\cdot \mathrm{cm}^{-2}\right)$ in initially highly $\mathrm{n}$-doped graphene $\left(4 \cdot 10^{12}\right.$ electrons $\left.\cdot \mathrm{cm}^{-2}\right)$. The effect of corona discharge is reversible, allowing $\mathrm{p}$ and $\mathrm{n}$ doping depending on the charge of ionic species deposited on the dielectric material. The method can be employed to perform electronic characterization of graphene and other 2D systems, such as 2DEGs at semiconductor interfaces or in topological insulators, and in some applications where fixed doping of $\mathrm{SiC} / \mathrm{G}$ is required, for example, quantum resistance metrology.

${ }^{1}$ K. Novoselov, A. Geim, S. Morozov, D. Jiang, M. Katsnelson, I. Grigorieva, S. Dubonos, and A. Firsov, Nature 438, 197 (2005).

${ }^{2}$ K. S. Novoselov, V. I. Fal'ko, L. Colombo, P. R. Gellert, M. G. Schwab, and K. Kim, Nature 490, 192 (2012).

${ }^{3}$ C. Virojanadara, M. Syväjarvi, R. Yakimova, L. I. Johansson, A. A. Zakharov, and T. Balasubramanian, Physical Review B 78, 245403 (2008).

${ }^{4}$ Y.-M. Lin, C. Dimitrakopoulos, K. A. Jenkins, D. B. Farmer, H.-Y. Chiu, A. Grill, and P. Avouris, Science 327, 662 (2010).

${ }^{5}$ V. Panchal, D. Cox, R. Yakimova, and O. Kazakova, Magnetics, IEEE Transactions on 49, 97 (2013).

${ }^{6}$ S. Kopylov, A. Tzalenchuk, S. Kubatkin, and V. I. Fal'ko, Applied Physics Letters 97, 112109 (2010).

${ }^{7}$ T. J. B. M. Janssen, A. Tzalenchuk, R. Yakimova, S. Kubatkin, S. Lara-Avila, S. Kopylov, and V. I. Fal'ko, Physical Review B 83, 233402 (2011).

${ }^{8}$ T. J. B. M. Janssen, J. M. Williams, N. E. Fletcher, R. Goebel, A. Tzalenchuk, R. Yakimova, S. Lara-Avila, S. Kubatkin, and V. I. Fal'ko, Metrologia 49, 294 (2012).

${ }^{9}$ J. S. Moon, D. Curtis, S. Bui, M. Hu, D. K. Gaskill, J. L. Tedesco, P. Asbeck, G. G. Jernigan, B. L. VanMil, R. L. Myers-Ward, C. R. Eddy, Jr., P. M. Campbell, and X. Weng, IEEE Electron Device Letters 31, 260 (2010).

${ }^{10}$ C. Riedl, C. Coletti, T. Iwasaki, A. Zakharov, and U. Starke, Physical review letters 103, 246804 (2009).

${ }^{11}$ E. Pallecchi, M. Ridene, D. Kazazis, C. Mathieu, F. Schopfer, W. Poirier, D. Mailly, and A. Ouerghi, Applied Physics Letters 100, 253109 (2012).

${ }^{12}$ D. Waldmann, J. Jobst, F. Speck, T. Seyller, M. Krieger, and H. B. Weber, Nat Mater 10, 357 (2011).

${ }^{13}$ C. Coletti, C. Riedl, D. S. Lee, B. Krauss, L. Patthey, K. von Klitzing, J. H. Smet, and U. Starke, Physical Review B 81, 1 (2010).

${ }^{14}$ S. Lara-Avila, K. Moth-Poulsen, R. Yakimova, T. Bjørnholm, V. Fal'ko, A. Tzalenchuk, and S. Kubatkin, Advanced Materials 23, 878 (2011).

${ }^{15}$ L. B. Loeb, ed., Electrical Coronas - Their Basic Physical Mechanisms (University of California Press, Berkeley and Los Angeles, 1965).

${ }^{16}$ D. Schroder, M. Fung, R. Verkuil, S. Pandey, W. Howland, and M. Kleefstra, Solid-State Electronics 42, 505 (1998).

${ }^{17}$ R. Williams and M. Woods, Journal of Applied Physics 44, 1026 (1973).

${ }^{18} \mathrm{R}$. Williams and A. Willis, Journal of Applied Physics 39, 3731 (1968).

${ }^{19}$ R. B. Comizzoli, Journal of The Electrochemical Society 134, 424 (1987).

${ }^{20}$ T. Yager, A. Lartsev, S. Mahashabde, S. Charpentier, D. Davidovikj, A. Danilov, R. Yakimova, V. Panchal, O. Kazakova, A. Tzalenchuk, S. Lara-Avila, and S. Kubatkin, Nano Letters 13, 4217 (2013).

${ }^{21}$ J. H. Park, D. Hwang, J. Lee, S. Im, and E. Kim, Thin Solid Films 515, 4041 (2007).

${ }^{22}$ N. B. Ukah, J. Granstrom, R. R. Sanganna Gari, G. M. King, and S. Guha, Applied Physics Letters 99, 243302 (2011). 